\title{
Study on 3D CFBG Vibration Sensor and Its Application
}

\author{
Qiuming NAN ${ }^{1,2^{*}}$ and Sheng $\mathrm{LI}^{1,2}$ \\ ${ }^{1}$ National Engineering Laboratory for Fiber Optic Sensing Technology, Wuhan University of Technology, Wuhan, \\ 430070, China \\ ${ }^{2}$ Key Laboratory of Fiber Optic Sensing Technology and Information Processing, Ministry of Education, Wuhan, \\ University of Technology, Wuhan, 430070, China \\ *Corresponding author: Qiuming NAN_E-mail: 197114012@qq.com
}

\begin{abstract}
A novel variety of three dimensional (3D) vibration sensor based on chirped fiber Bragg grating (CFBG) is developed to measure 3D vibration in the mechanical equipment field. The sensor is composed of three independent vibration sensing units. Each unit uses double matched chirped gratings as sensing elements, and the sensing signal is processed by the edge filtering demodulation method. The structure and principle of the sensor are theoretically analyzed, and its performances are obtained from some experiments and the results are as follows: operating frequency range of the sensor is $10 \mathrm{~Hz}-500 \mathrm{~Hz}$; acceleration measurement range is $2 \mathrm{~m} \cdot \mathrm{s}^{-2}-30 \mathrm{~m} \cdot \mathrm{s}^{-2}$; sensitivity is about $70 \mathrm{mV} / \mathrm{m} \cdot \mathrm{s}^{-2}$; crosstalk coefficient is greater than $22 \mathrm{~dB}$; self-compensation for temperature is available. Eventually the sensor is applied to monitor the vibration state of radiation pump. Seen from its experiments and applications, the sensor has good sensing performances, which can meet a certain requirement for some engineering measurement.
\end{abstract}

Keywords: Three-dimensional (3D); matched; chirped fiber Bragg grating (CFBG); edge filtering demodulation; crosstalk

Citation: Qiuming NAN and Sheng LI, "Study on 3D CFBG Vibration Sensor and Its Application," Photonic Sensors, 2016, 6(1): 90-96.

\section{Introduction}

Vibration measurement is one of the most effective methods for mechanical equipment condition monitoring and fault diagnosis. However, vast majority of vibration measurement is based on electromagnetic sensors, although this technology has been used for some of its advantages, its application is greatly limited in some special occasion [1-4]. For example, in the flammable, explosive, and electromagnetic-interference situation, a large network is required, and the signal is required to transmit over a long distance. The fiber Bragg grating sensing technology is a newly minted one, which has such characteristics, such as nonelectric detection, anti-interference of electromagnetic, ease to use in large-scale network, large amount of information, and long-distance transmission. It provides a new technical means for the vibration monitoring of mechanical equipment $[5,6]$. According to statistics, there are being done some researches on a one-way fiber grating vibration sensor [7], but in practical application, it is frequently demanded to detect the spatial vibration of a certain part of the machine at the same time. Due to the limitation of the installation space or 
other reasons, three one-way vibration sensors can be installed in the identical position so difficultly that the authentic state of equipment can not be monitored completely and correctly [8-10]. Consequently, it is very essential to conduct the research on a three-dimensional chirped fiber Bragg grating (3D CFBG) vibration sensor.

\section{Structure and principle of sensor}

\subsection{Structure design}

According to the application requirements, the overall design goals of the sensor are as follows:

(1) Integration and seal design

(2) Working frequency $\geq 500 \mathrm{~Hz}$

(3) Acceleration range $\geq 20 \mathrm{~m} \cdot \mathrm{s}^{-2}$

(4) Ambient temperature range: $-20^{\circ} \mathrm{C}-80^{\circ} \mathrm{C}$

(5) Single dimensional size $\leq 100 \mathrm{~mm}$

According to this goal, we design a structure of the three-dimensional fiber grating vibration sensor as shown in Fig. 1. The sensor is composed of three unidirectional sensing units that are mutually vertical. Each sensing unit is composed of a base, an elastomer, a mass block, and two CFBGs, and its structure and principle are shown in Fig. 2. Two CFBGs written in the same optic fiber are formed into precise matching by accurate fabrication process, and they are symmetrically fixed between the base and the mass block. In order to avoid dead zone, we give them $2 \mathrm{~nm} / \mathrm{s}$ pretension. When the sensor receives the vibration signal from the outside world, the mass supported by the elastic membrane will vibrate up and down to drive the CFBGs stretch along the axial direction, and the reflection peaks of CFBG1 and CFBG2 will change, as shown in Fig. 3. The light emitting diode (LED) bandwidth is approximately $40 \mathrm{~nm}$. The detected signal by the photodetector is the change in the envelope spectrum of the two CFBGs, which is negatively correlated with that of the overlapped spectrum. To take the upward acceleration of the mass block as an example, CFBG2 elongates, CFBG1 shrinks, the overlapped area becomes bigger but the detected signal is negative, and vice versa.

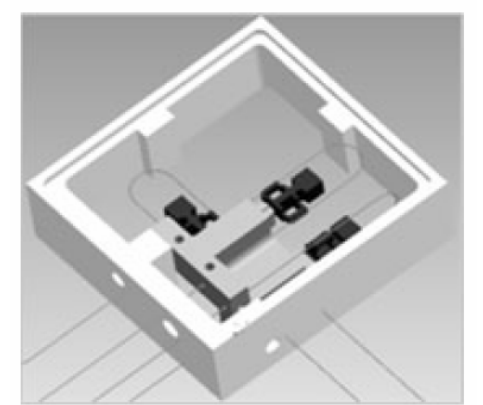

Fig. 1 Structure of 3D CFBG accelerometer.

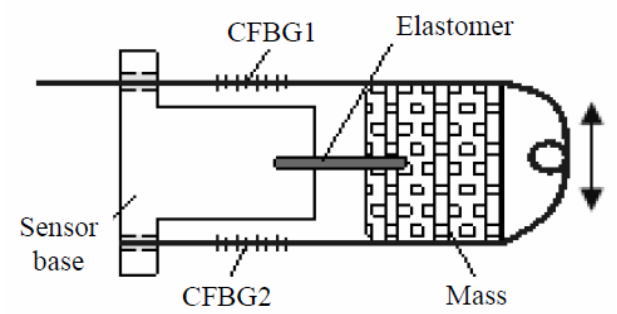

Fig. 2 Structure and principle of sensing unit.

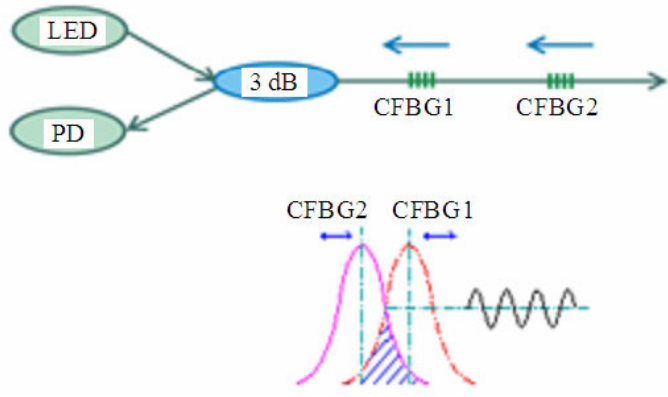

Fig. 3 Principle of matching demodulation.

From the structure and principle of the sensor, the sensor has the following two notable advantages. Firstly, when the external temperature changes, the reflection spectra of the two gratings will move to the same direction, and the envelope area remains constant, so the self-compensation for temperature change can be realized. Secondly, when the sensor is forced to vibrate, the moving direction of two reflection spectrums are opposite, and the envelope area is increased significantly. Accordingly, the sensitivity of the sensor is effectively improved.

\subsection{Theoretical analysis}

In order to further illustrate the working principle of the sensor, the authors have conducted theoretical calculation. As shown in Fig. 4, the upward exciting force exerted to the mass block is $F_{a}=M a$, and it makes the elastomer deform, while CFBG2 is elongated to generate a left tension $F_{f}$. 
For the elastomer, the stress state can be transformed into equivalent to the force $F_{a}$ and a clockwise torque $M=F_{f} \times d$, where $d$ is the distance between mass block and elastic node, namely the distance between $A$ and $B$ in Fig. 4.

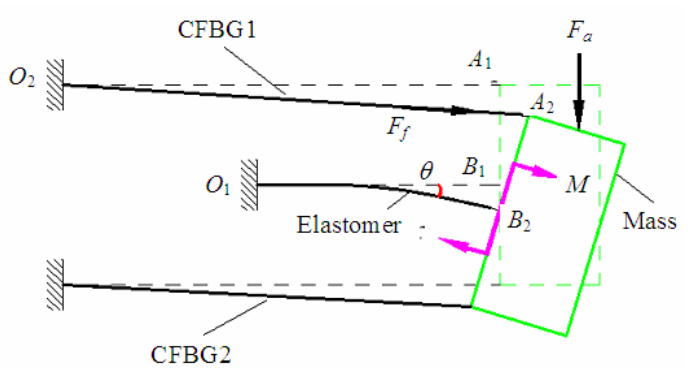

Fig. 4 Schematic diagram of the force exerted to the sensor.

According to the calculation of mechanics of materials for the deformation of bending beam, the deflection $\omega$ of elastic body can be expressed as

$$
\omega=\frac{F_{a} l^{3}}{3 E I}-\frac{M l^{2}}{2 E I}
$$

where $l$ is the length of the elastomer, $E$ is the elastic modulus, and $I$ is the moment of inertia.

$\Delta L=O_{2} A_{2}-O_{2} A_{1}$

$=\sqrt{\left(O_{2} A_{1}+A_{2} B_{2} \sin \theta\right)^{2}+\left(A_{1} B_{1}+B_{1} B_{2}-A_{2} B_{2} \cos \theta\right)^{2}}$ $-O_{2} A_{1}$.

The tension $\Delta L$ of the optical fiber subjected to external vibration can be expressed as (2). As shown in Fig. 4, $\theta$ is the angle of the elastomer caused by the stress. As the rotation angle is very small, (2) can be expressed as

$$
\Delta L=\sqrt{L^{2}+\omega^{2}}-L=\omega
$$

where $L$ is the original length of the fiber.

The axial stress $\varepsilon$ of the fiber and the change $\Delta \lambda$ in wavelength caused by the stress can be expressed respectively as

$$
\varepsilon=\frac{\Delta L}{L}=F_{f} / E_{f} A
$$

where $E_{f}, A$, and $K_{f}$ are the elastic modulus, cross-sectional area, and stiffness of optical fiber, respectively.

$$
\Delta \lambda=\left(1-P_{e}\right) \lambda \varepsilon
$$

where $P_{e}$ and $\lambda$ are the effective elastic optical coefficient and initial wavelength of fiber grating, respectively.

From (1) to (5), the sensitivity $S$ of the sensor can be expressed as

$$
S=\frac{\Delta \lambda}{a}=\left(1-P_{e}\right) \lambda \frac{1}{L} \frac{2 m l^{2}}{6 E I+3 K_{f} d l^{2}}
$$

where $m$ is the quality of the mass. From (6), it can be observed that there is a linear relationship between the wavelength change $\Delta \lambda$ of FBG and the outside acceleration $a$.

The elastomer can be regarded as a uniform cantilever beam, so its stiffness $K_{e}$ is expressed as

$$
K_{e}=\frac{3 E I}{l^{3}} .
$$

According to the kinematics equation of the object structure, the first-order resonant frequency $f$ of the sensor is calculated as

$$
f=\frac{1}{2 \pi} \sqrt{\frac{K}{m}}=\frac{1}{2 \pi} \sqrt{\frac{K_{e}+(d / L)^{2} K_{f}}{m} .}
$$

From (6) and (8), if $m$ is larger and $l$ is longer, then $S$ is bigger, and $f$ is smaller; vice versa. Therefore, the structure parameters of the sensor should be reasonably designed according to the requirement of the measurement.

\section{Performance tests of sensor}

In accordance with the above designs, we have made some samples of 3D CFBG vibration sensor. Next, we test the sensing performances of the sensor by means of the experimental apparatus, as shown in Fig. 5. These sensing performances mainly include amplitude-frequency characteristics, acceleration characteristics, anti-crosstalk, and anti-temperature influence performance.

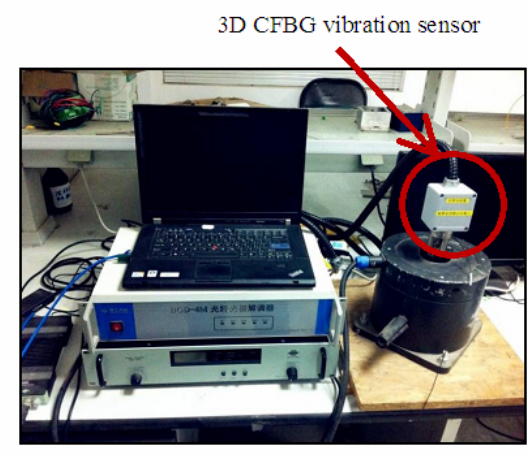

Fig. 5 Vibration testing system. 


\subsection{Amplitude-frequency characteristics}

In the amplitude-frequency characteristic test, the output acceleration of the exciter is kept constant, $3 \mathrm{~m} / \mathrm{s}^{-2}$ as the input vibration signal. The frequency of the input signal starts from $10 \mathrm{~Hz}$, incrementing $50 \mathrm{~Hz}$ as a step, and when the frequency value reaches $800 \mathrm{~Hz}$, the step size of the frequency is adjusted to $20 \mathrm{~Hz}$. The experimental data of each step should be recorded. During the test, the amplitude-frequency characteristics of three sensing units $(X, Y, Z)$ are measured, respectively, as shown in Fig. 6. The results show that their first-order resonant frequencies are $910 \mathrm{~Hz}, 890 \mathrm{~Hz}$, and $890 \mathrm{~Hz}$, respectively, the flat segments of the curves are all the range from $10 \mathrm{~Hz}$ to $500 \mathrm{~Hz}$, namely, and the operating frequency range of the sensor is $10 \mathrm{~Hz}-$ $500 \mathrm{~Hz}$.

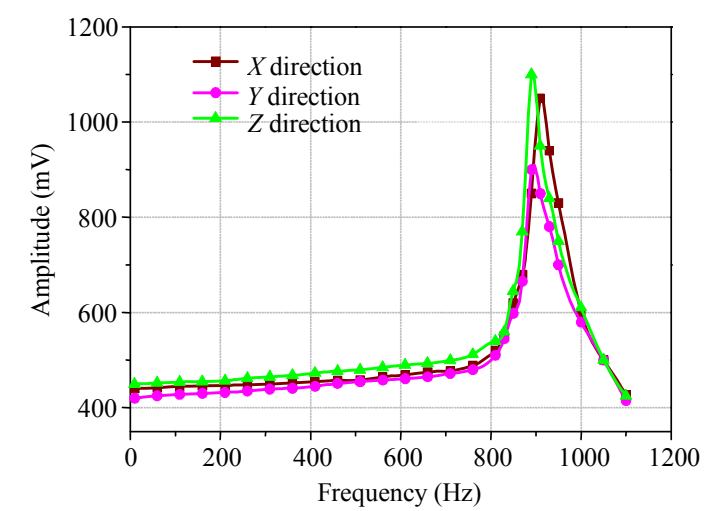

Fig. 6. Amplitude-frequency characteristics of 3 sensing units.

\subsection{Linear calibration}

Linear calibration is a good method to test the sensor's performances like sensitivity, linearity, and linear range. The frequency of the input signal is $200 \mathrm{~Hz}$, and measurement acceleration is within $2 \mathrm{~m} \cdot \mathrm{s}^{-2}-30 \mathrm{~m} \cdot \mathrm{s}^{-2}$, which are read out by the piezoelectric standard acceleration sensor, type 4371 . Write down the output voltage values of the 3D CFBG vibration sensor at each setting value. The calibration curves are shown in Fig.7. From Fig.7, we can see when the input acceleration is within the range of $2 \mathrm{~m} \cdot \mathrm{s}^{-2}-30 \mathrm{~m} \cdot \mathrm{s}^{-2}$, the linear degrees of calibration curves are all more than 0.999 , and the three sensitivities are $73 \mathrm{mV} / \mathrm{m} \cdot \mathrm{s}^{-2}, 69 \mathrm{mV} / \mathrm{m} \cdot \mathrm{s}^{-2}$, and $75 \mathrm{mV} / \mathrm{m} \cdot \mathrm{s}^{-2}$, respectively. With an increase in the acceleration, the output voltage increases slowly, and the sensitivity decreases. When the acceleration is more than $30 \mathrm{~m} \cdot \mathrm{s}^{-2}$, the output voltage becomes saturated. Therefore, the acceleration measurement range of the sensor can be considered as $2 \mathrm{~m} \cdot \mathrm{s}^{-2}-$ $30 \mathrm{~m} \cdot \mathrm{s}^{-2}$.

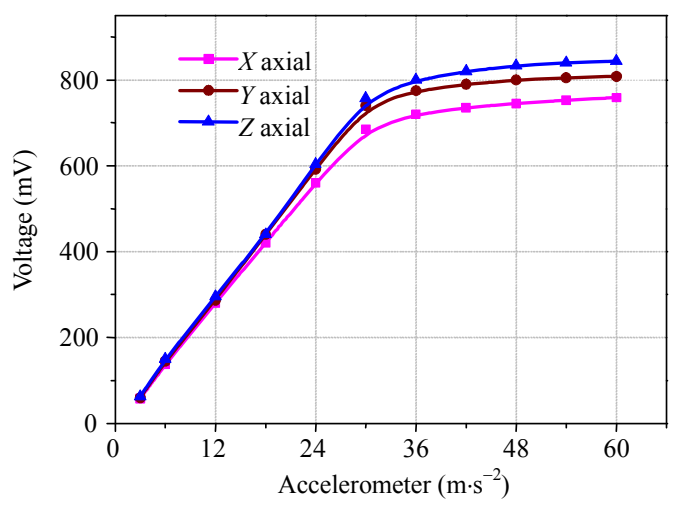

Fig. 7 Calibration curve of 3D FBG accelerometer.

\subsection{Anti-crosstalk performance}

First of all, let the exciter vibrate in the $X$ axis direction, the input signal is a sine wave signal, of which the vibration frequency is $60 \mathrm{~Hz}$, and the acceleration is $6 \mathrm{~m} \cdot \mathrm{s}^{-2}$. Three-direction response data are collected at the same time, and the effective values of output voltage are $450 \mathrm{mV}, 32 \mathrm{mV}$, and $34 \mathrm{mV}$, respectively. Similarly, under the condition of the invariable vibration signal, let $Y$ and $Z$ axes be the main vibration directions, respectively, repeat the above experiments, and the results are shown in and Figs. 8, 9, and 10. From Table 1, the maximum crosstalk coefficient can be calculated about $22 \mathrm{~dB}$, which indicates that crosstalk has little effect on the measurement.

Table 1 Test results of crosstalk coefficient (mV).

\begin{tabular}{ccccc}
\hline \multicolumn{2}{l}{ Main vibration direction } & $X$ & $Y$ & $Z$ \\
\hline \multirow{2}{*}{ Output } & $X$ & 450 & 36 & 38 \\
response & $Y$ & 32 & 462 & 39 \\
& $Z$ & 34 & 35 & 470 \\
\hline
\end{tabular}

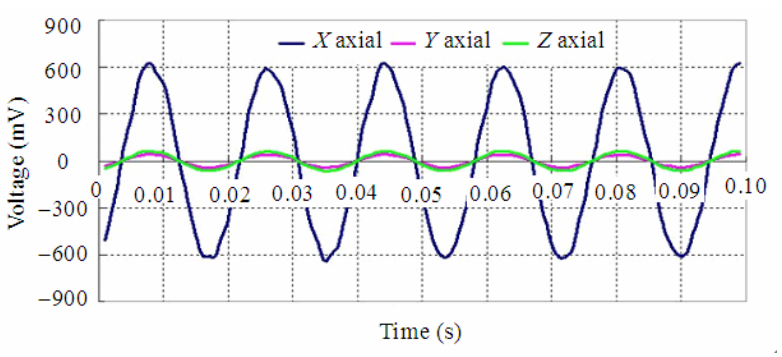

Fig. 8 Response curves of $X$ shaft as main vibrating direction. 


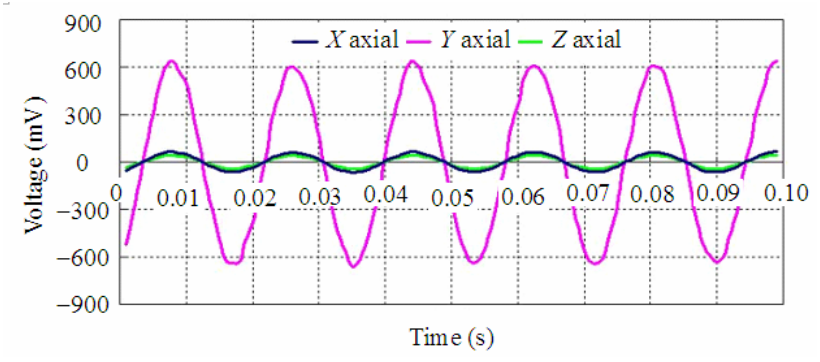

Fig. 9 Response curves of $Y$ shaft as main vibrating direction.

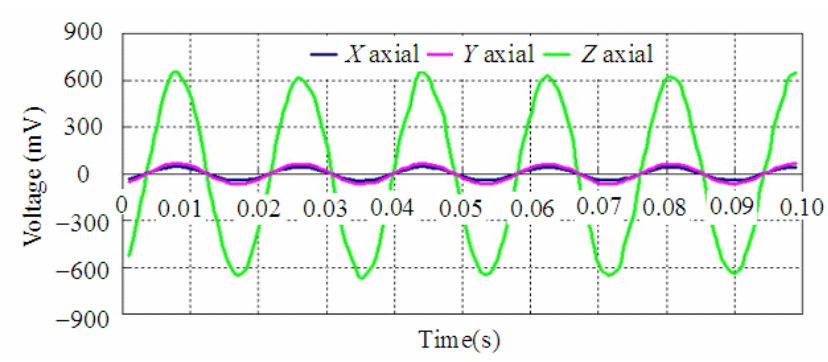

Fig. 10 Response curves of $Z$ shaft as main vibrating direction.

\subsection{Anti-temperature performance}

The sensing performance of the sensor based on edge filtering demodulation is largely determined by the matching state of the two gratings. When the ambient temperature changes, the reflection peaks of the two gratings will shift; if the two reflection peaks move synchronously, the matching state keeps constant, but if they are not synchronized, the matching state will change. The results of the anti-temperature influence experiment are shown in Fig. 11.

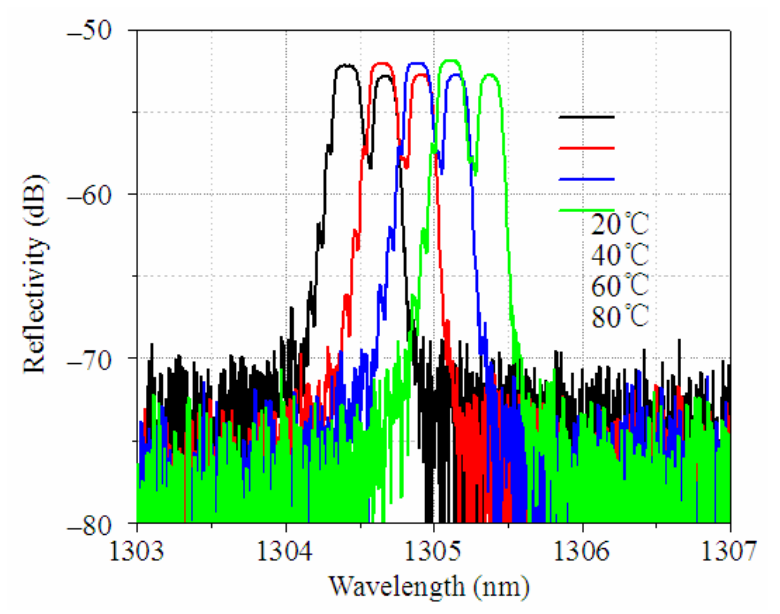

Fig. 11 Matching state of double gratings.
From Fig. 11, it can be seen that the matching state of the double gratings has no change when the temperature varies from $20^{\circ} \mathrm{C}$ to $80^{\circ} \mathrm{C}$, and the output is stable, which shows that the sensor is insensitive to the change of the temperature and has a strong ability to resist temperature interference.

\section{Application}

\subsection{Application description}

The 3D CFBG vibration sensor developed in the paper has been used in the vibration monitoring of the radiation pump in petrochemical industry. In the industrial test, 3 pumps were monitored and 2 sensors were installed on each pump. The monitoring parameters included the vibration of the bearing seat in the three directions, the horizontal $(V)$, vertical $(H)$, and axial $(A)$. In the installation, the sensor was installed on the bearing seat by bolts. Site installation conditions are shown in Fig. 12.

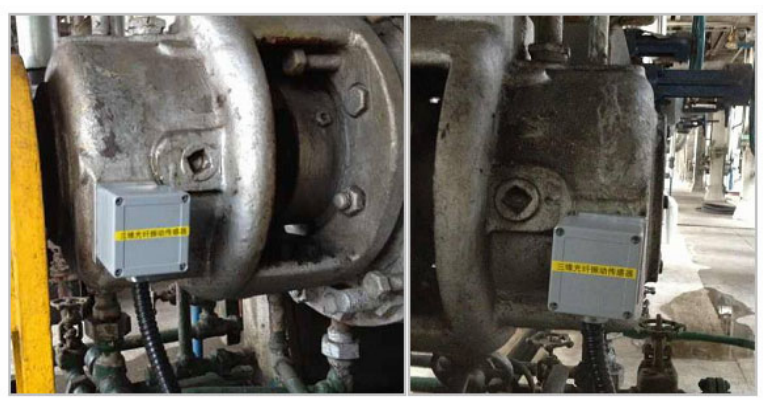

Fig. 12 Sensor site installation photos.

\subsection{Application results}

On Jan 20th, 2014, the online monitoring system issued a warning signal. It was found through the query that the vibration speed of $3 \mathrm{H}$ of the No.3 pump was about $5.2 \mathrm{~mm} / \mathrm{s}$, as shown in Fig. 13, and this value was increasing. Further analysis of the vibration data in other directions, which were larger than the normal values, is shown in Fig. 14, which showed that the working condition of the pump was deteriorating. Moreover, further studies found that there were the fault characteristic frequencies of the inner ring and cage of the bearing in the velocity 
spectrum. Through the comprehensive analysis of the above test results, the preliminary diagnosis was that there were peeling defect on the inner ring and severe wear on the cage.

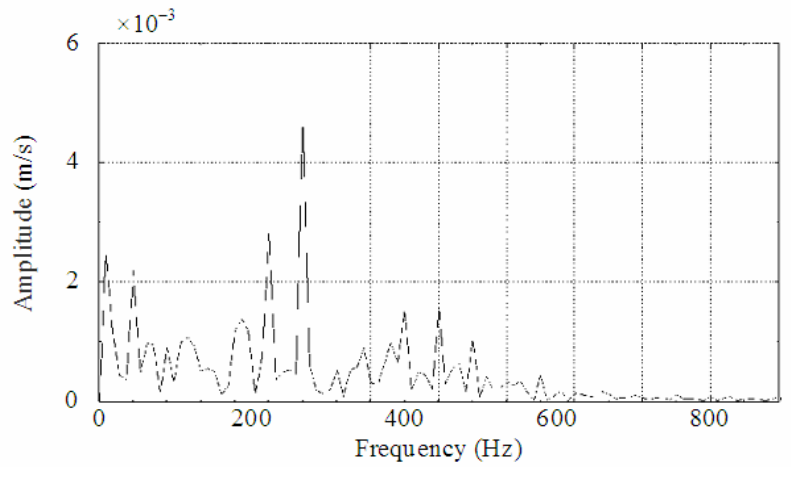

Fig. 13 Frequency spectrum of $3 \mathrm{H}$ measuring point.

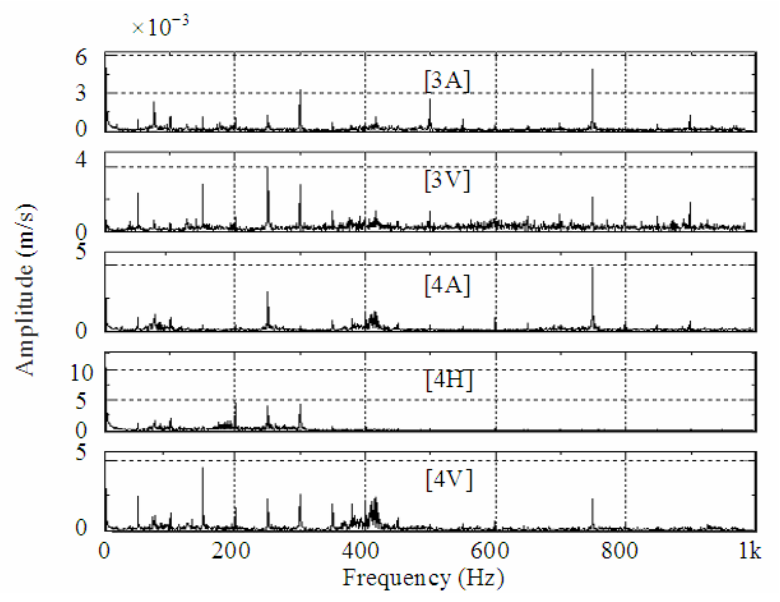

Fig. 14 Frequency spectrum of multimeasuring points.
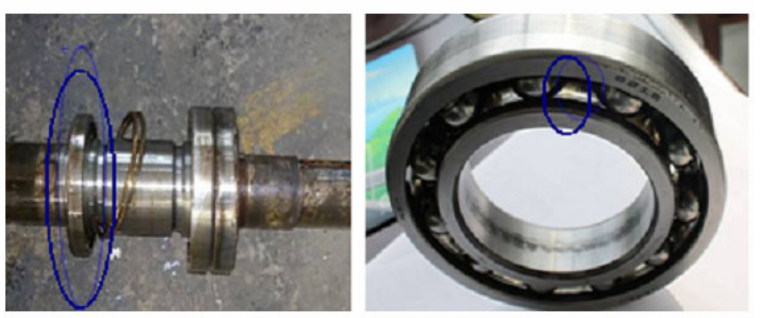

Fig. 15 Fault bearing physical picture.

On Jan 23th, the radiation pump was removed for maintenance and it was found that there were two larger and a dozen smaller exfoliations on the inner ring of the bearing, and some obvious damages on the surface of the shaft, as shown in Fig. 15. According to the above situation, the bearing was replaced and the system showed the pump had been into working in the normal state after maintenance, which adequately demonstrated the accuracy and reliability of the above fault diagnosis.

\section{Conclusions}

A novel variety of $3 \mathrm{D}$ vibration sensor based on CFBG is developed to measure $3 \mathrm{D}$ vibration in the mechanical equipment field. Theoretical analysis and experimental tests have been performed for the sensor, and it has been applied to engineering practice. The main conclusions are as follows.

(1) In order to have a good performance, the following innovative design is implemented. Firstly, the sensing unit is designed as an improved cantilever structure to increase the operating frequency of the sensor. Secondly, the two matched chirped gratings are used as a sensing element to increase the measurement range of acceleration. Finally, the demodulation method based on the edge filtering is used to solve the problem of high frequency signal acquisition.

(2) The results of performance test are as follows. The operating frequency range of the sensor is $10 \mathrm{~Hz}$ - $500 \mathrm{~Hz}$, the acceleration measurement range is $2 \mathrm{~m} \cdot \mathrm{s}^{-2}-30 \mathrm{~m} \cdot \mathrm{s}^{-2}$, the sensitivity is about $70 \mathrm{mV} / \mathrm{m} \cdot \mathrm{s}^{-2}$, the crosstalk coefficient is greater than $22 \mathrm{~dB}$, and it has a strong ability to resist the temperature interference. These experimental results are in good agreement with the theoretical analysis.

(3) The 3D CFBG vibration sensor has been applied to monitor the working state of the radiation pump and the process of the bearing fault has been successfully monitored, which may be an important basis for fault analysis.

\section{Acknowledgment}

This research was funded by the Key Project of National Science Foundation of China, Award Number: 61290311.

Open Access This article is distributed under the terms of the Creative Commons Attribution 4.0 International License (http:// creativecommons.org/licenses/by/4.0/), 
which permits unrestricted use, distribution, and reproduction in any medium, provided you give appropriate credit to the original author(s) and the source, provide a link to the Creative Commons license, and indicate if changes were made.

\section{References}

[1] Y. Zheng, "Vibration monitoring and analysis for rotating machinery," Gas Tribine Technology, 2010, 23(1): 39-48.

[2] D. Fu and Y. Wang, "The research of on-line vibration monitoring system of rotating machinery," Machine Tool \& Hydraulics, 2005, (1): 152-153.

[3] Q. M. Nan, "Study on dynamic sensing and monitoring methods for petrochemical facilities based on optical fiber grating," Ph.D. dissertation, Dept. Wuhan University, China, 2014.

[4] Z. Zhou, Q. Liu, Q. Ai, and C. Xu, "Intelligentmonitoring and diagnosis for modern mechanical equipment based on the integration of embedded technology and FBGS technology," Measurement, 2011, 44(9): 1499-1511.
[5] Q. M. Nan, "Study and application of CFBG vibration sensor with symmetrical push-pull configuration," in the 22nd International Conference on Optical Fiber Sensor, Beijing, China, 15-19, 2012.

[6] G. Wang and B. Xie, "Improving the performance of chirped fiber grating with cladding being etched as sinusoidal function," Optik International Journal for Light and Electron Optics, 2010, 122(6): 557-559.

[7] Y. Zhao and Z. Li, "Study on tri-axial accelerometer based on FBG," Chinese Journal of Scientific Instrument, 2006, 27(6): 299-301.

[8] J. Wang, C. Chen, D. Tang, C. Zhang, and Y. Cui, "Three-component photo-elastic fiber optic accelerometer based on the photo-elastic effect," Chinese Journal of Sensors and Acuators, 2006, 19(3): 804-806.

[9] L. Ding, J. Zhao, J. Wang, and Q. Sui, "Design of FBG vibration sensor based on matching filter demodulation," Electro-Optic Technology Application, 2009, 24(3): 36-39.

[10] Q. Nan, "Research on 3D FBG Accelerometer and demodulation method," Chinese Optics Letters, 2014, (A01): 149-153. 